\title{
Change in Mechanical Properties of Ion-Irradiated Ceramics Studied by Nanoindentation*1
}

\author{
Shinsuke Nakano ${ }^{1, * 2}$, Shunsuke Muto ${ }^{1}$ and Tetsuo Tanabe ${ }^{2}$ \\ ${ }^{1}$ Department of Materials, Physics and Energy Engineering, Nagoya University, Nagoya 464-8603, Japan \\ ${ }^{2}$ Department of Advanced Energy Engineering Science, Interdisciplinary Graduate School of Engineering Science, \\ Kyushu University, Fukuoka 812-8581, Japan
}

Changes in hardness of several representative ceramics and semiconductors associated with ion irradiation were systematically studied using a combination of nanoindentation and finite element analysis. We established a new method for obtaining the precise hardness of the embedded damaged layer of ion-irradiated samples. The method was applied to silicon carbide, $\alpha$-quartz, silica glass and silicon, which enabled us to semi-quantitatively discuss changes in their mechanical properties with irradiation-induced structural changes on the basis of experimentally obtained material parameters. Finally, we propose a new atomistic mechanism for plastic deformation of covalent amorphous materials. The present results will provide a standard framework for discussing mechanical property changes in ceramics with energetic particle irradiation.

(Received September 20, 2005; Accepted November 14, 2005; Published January 15, 2006)

Keywords: nanoindentation, finite element method, hardness, $\mathrm{SiO}_{2}$, silicon, silicon carbide, ion irradiation

\section{Introduction}

To develop new structural materials resistant to long exposure to energetic particle irradiation, such as those used in nuclear fusion and fission reactors, mechanical property data are required, essential for determining the reactor lifetime. The long accumulation of knowledge from both theory and experiments has enabled us to understand to a considerable degree how lattice defects influence elastic and plastic properties in metals and alloys. ${ }^{1)}$ However, it has not yet been fully established whether one can adopt the dislocation theories developed for metals to covalent or ionic ceramics as they stand, or systematically understand how irradiation damages affect their mechanical properties.

Nowadays, integration of multi-functions accelerates downsizing of the constituent elements in developing novel materials, and it is getting increasingly more difficult and essential to measure the physical and chemical properties of the constituent parts. Mechanical properties, in particular, Young's modulus, yield stress and hardness are intrinsically macroscopic quantities, and thus conventional methods are not always applicable to thin films, fine particles, and other nanomaterials.

The nanoindentation method is used for measuring the mechanical response of materials by pressing a sharp diamond indenter into their surface in a well-controlled manner. ${ }^{2)}$ The experimental techniques and analysis methods involved have advanced dramatically, allowing control of the test area and indentation depth at the submicron and nanometer scales, respectively. Thus, nanoindentation has become well-suited for measuring mechanical properties in small areas and thin films. However, when measuring the mechanical properties of a thin film grown on a substrate, the

\footnotetext{
*1 This Paper was Originally Published in Japanese in J. Japan Inst. Metals 69 (2005) 815-824.

${ }^{* 2}$ Graduate Student, Nagoya University. Present address: Mitsubishi Heavy Industries, Ltd.
}

measured parameters should be ascribed to the film-substrate composite even though the maximum indentation depth may be set to be less than the film thickness, since the overall elastic response is inevitably affected by the elasto-plastic deformation of the substrate. ${ }^{2)}$ In particular, the mechanical properties of a hard ultra-thin film may be smeared out by the softer substrate properties. Thus, the mechanical properties of thin films are obtained by reproducing the nanoindentation test in a computer with a finite element method (FEM), taking into account the elasto-plastic properties of both materials of interest, the diamond indenter and the elastic rigidity of the testing instrument. The materials parameters of the substrate are independently determined in advance and those of the thin film on it are determined by matching the simulated load-displacement (L-D) curve with the experimental one. ${ }^{3)}$ This method considerably improves the accuracy of the mechanical parameters estimation, as compared to the conventional method, ${ }^{4)}$ where the parameters are estimated by utilizing a small portion of the obtained L-D curve.

To investigate mechanical property changes upon energetic particle irradiation, neutron irradiation is used as it is the most suitable method for introducing defects into the material of interest in a uniform manner. However, a nuclear reactor for neutron irradiation present difficulties in controlling irradiation temperature and fluence, and also, the machine time available is limited. By contrast, ion irradiation is capable of easily controlling temperature and other conditions, though the irradiation damage is distributed around a certain depth from the surface and it is difficult to directly measure the mechanical properties at a particular dose. Nanoindentation tests along the depth direction on a cross-section sample would provide information on the depth distribution or fluence dependence of mechanical properties ${ }^{5)}$ but it is not realistic to measure the properties of a region localized at the submicron scale, because the residual impression ( $>$ a few hundred nanometers) limits the spatial resolution of the test.

Keeping the above in mind, we first establish an analysis 
method for nanoindentation to precisely measure and obtain the mechanical parameters of the damaged layer embedded within the localized region in the sample. This method is then applied to $\alpha$-quartz and silica glass, whose hardness deviates from the theoretically predicted values, and also to silicon carbide and silicon as representative covalent materials for comparison. Their hardness is measured as a function of irradiation dose, and a phenomenological model for explaining the experimental data is proposed. Finally, the plastic deformation mechanisms of the materials are discussed on the basis of the parameters derived by the present analyses.

\section{Analysis Method for Obtaining the Mechanical Parameters of an Ion-Irradiation-Damaged Layer by Nanoindentation Tests and FEM (NI-FEM)}

We applied a method developed by Knapp et al. ${ }^{3)}$ (outlined in Appendix 1) to the present problem. In the present study, the stress-strain behavior was assumed to be that of a perfect elasto-plastic body (see Fig. 1) since the materials of interest are covalent or ionic materials. The diamond indenter was treated as being elastic. We found that the changes in the Poisson's ratio and work-hardening coefficient had no influence on the simulation results. Thus, the only parameters characterizing the mechanical properties of the samples were

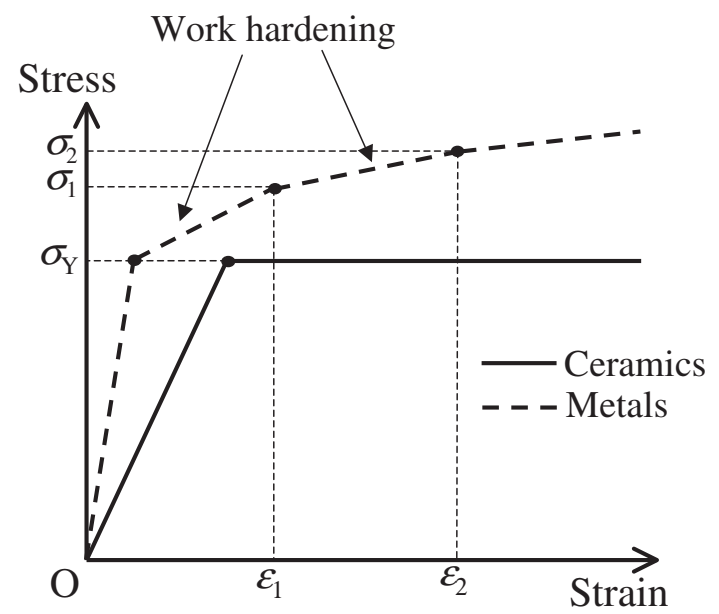

Fig. 1 Elasto-plastic model used in the present nanoindentation simulations by FEM. the Young's modulus, $E$, and the critical yield stress, $Y$. This model is thought to be reasonable because all the experimental L-D curves were reproduced by the computer simulations within the present experimental accuracy. We confirmed that the simulation results showed no variation with the contact friction coefficient between the sample and indenter from 0 to unity. Hence, this coefficient was set to 0.2 .

As mentioned in the Introduction, ion irradiation inevitably involves a depth distribution of irradiation damage, and thus, it is not possible for an indentation test conducted on the surface to measure the absolute hardness at a particular irradiation dose. We applied a model that divides the ionirradiated sample into multiple layers along the normal to the surface, as schematized in Fig. 2. First, the nominal damage distribution is calculated, using the SRIM code ${ }^{6}$ [Fig. 2(b)]. Then, a sample irradiated at the lowest dose possible is tested by nanoindentation. For this experiment, a PC model sample is divided into two, a damaged and a non-damaged layer, as shown in Fig. 2(a). The mechanical parameters, $E_{0}$ and $Y_{0}$, of the unirradiated sample are determined in advance by the NIFEM method. The pair of parameters $\left(E_{1}, Y_{1}\right)$ for the damaged layer can be determined by substituting the $\left(E_{0}, Y_{0}\right)$ for the non-damaged layer and simulating the indentation test. The $\left(E_{1}, Y_{1}\right)$ determined are assigned to the material irradiated at the dose averaged over the layer. Next, a second nanoindentation measurement is carried out on a sample with a slightly increased irradiation dose. This time, the model sample for the simulation is divided into four layers: lightly damaged, damaged, lightly damaged and nondamaged, from surface to bottom, as shown in Fig. 2(c). The thickness of the lightly damaged layers is determined so that the averaged dpa coincides with that of the damaged layer in the previous step. Hence, $(E, Y)$ of the lightly damaged layer can be assigned as $\left(E_{1}, Y_{1}\right)$ of the damaged layer in the previous step, so that the only parameters to be determined are the pair $\left(E_{2}, Y_{2}\right)$ of the most damaged layer. By repeating this procedure, $\left(E_{2}, Y_{2}\right)$ of the most damaged layer is determined in each iteration. It is possible to divide the model into more than four layers from the third step on, but we confirmed that a model with more than four layers provided no improvement in accuracy.

After obtaining sets of $(E, Y)$ as functions of the irradiation dose, the theoretical L-D curve can be plotted by simulating

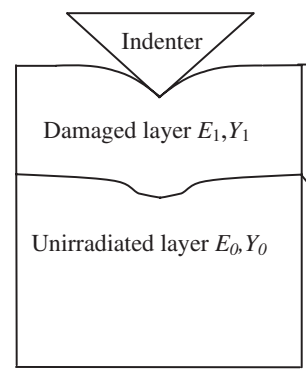

(a)

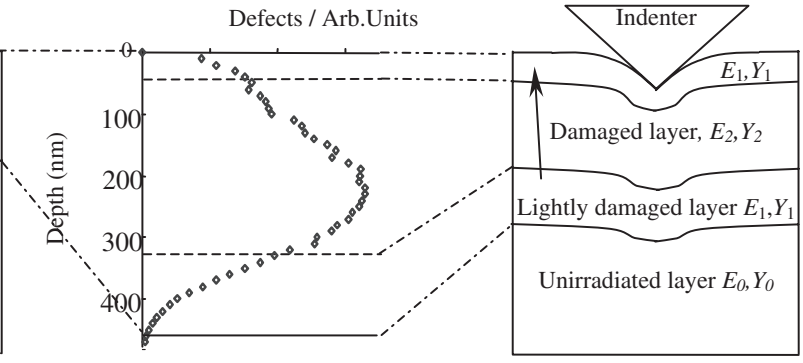

(b) (c)

Fig. 2 Schematic of the multi-layer model for deriving the mechanical parameters $E$ and $Y$ of the embedded damaged layer. (a) Initial two-layer model. The sample is divided into a damaged layer and an unirradiated layer. (b) Defect distribution derived by the TRIM code. ${ }^{6}$ The dpa (displacements per atom) value of each layer is estimated by averaging the dpa distribution over the thickness of the layer. (c) Four-layer model for successive steps. $E_{2}$ and $Y_{2}$ can be estimated using the $E_{0}, E_{1}, Y_{0}, Y_{1}$ determined in the previous step. 
the ideal nanoindentation test on the virtual sample having the derived $(E, Y)$ at each irradiation dose. Finally, the hardness $H$ is calculated on the resultant L-D curve using the Oliver-Pharr method ( $c f$. eq. (A-1) in Appendix 1). The conventional analysis applies eq. $(\mathrm{A} \cdot 1)$ to an experimental $\mathrm{L}-$ D curve, which limits the accuracy of the parameters to errors of above $10 \%$. Because of the experimental scatter in the maximum load, $F$, and the initial slope of the unloading curve, $S$, as well as the inaccuracy in the projected contact area, $A$. The present method estimates $(E, Y)$ using the L-D curve averaged over many trials, and conducts an ideal nanoindentation test on a computer, which eliminates the uncertainties in $F, S$, and $A$. The accuracy of the hardness, $H$, is determined on the basis of the accuracies of $(E, Y)$, usually to within $5 \%$. We discuss the validity of the multi-layer model in Section 4.2.

\section{Experimental}

The samples used are $\alpha$-SiC $(6 \mathrm{H},(0001)$-oriented), $\alpha$-quartz single crystals and fused silica glass, the sizes of which are listed in Table 1.

To introduce defects into the samples, helium ions $\left(\mathrm{He}^{+}\right)$ are irradiated at room temperature at an accelerating voltage of $20 \mathrm{kV}$. The defect distribution in the silica glass calculated with the SRIM code is shown in Fig. 2(b), and the damage peak is located at $100-200 \mathrm{~nm}$ from the surface in all samples. $\mathrm{He}^{+}$irradiation may induce bubble formation upon prolonged irradiation, in which case the experimental L-D curve would exhibit a characteristic profile associated with bubble formation, and hence, the present simulation would no longer reproduce the experimental L-D curve. This situation did not arise in the present study, and thus, the possibility of bubble formation is ruled out.

Silicon single crystals were also irradiated with hydrogen ions $\left(\mathrm{H}^{+}\right)$and $\mathrm{He}^{+}$for comparison, and the $\mathrm{He}^{+}$-irradiated sample showed L-D curves characteristic of bubble formation. Thus, we excluded the results of $\mathrm{He}^{+}$-irradiated silicon in the present report.

In nanoindentation measurements, the maximum indentation depth was set to be approximately $100 \mathrm{~nm}$, and 20 tests were conducted for each irradiation dose. Of the $20 \mathrm{~L}-\mathrm{D}$ curves obtained, those showing some anomaly due to the different surface conditions were eliminated, and the rest were averaged.

\section{Experimental Results}

\subsection{Irradiation dose dependence of mechanical param- eters}

Changes in the hardness $H$ of $\mathrm{SiC}$ determined by the conventional method and the present NI-FEM multi-layer

Table 1 Samples and irradiation conditions used in the present study.

\begin{tabular}{cccc}
\hline Sample & Size $[\mathrm{mm}]$ & Implant ion & Accel. Volt. [keV] \\
\hline$\alpha$-SiC $(6 \mathrm{H})$ & $3 \times 3 \times 0.5$ & $\mathrm{He}^{+}$ & 20 \\
$\mathrm{Si}$ & $3 \times 3 \times 0.5$ & $\mathrm{H}^{+}$ & 13 \\
$\alpha$-quartz & $13 \phi \times 1$ & $\mathrm{He}^{+}$ & 20 \\
Silica (fused) & $13 \phi \times 2$ & $\mathrm{He}^{+}$ & 20 \\
\hline
\end{tabular}

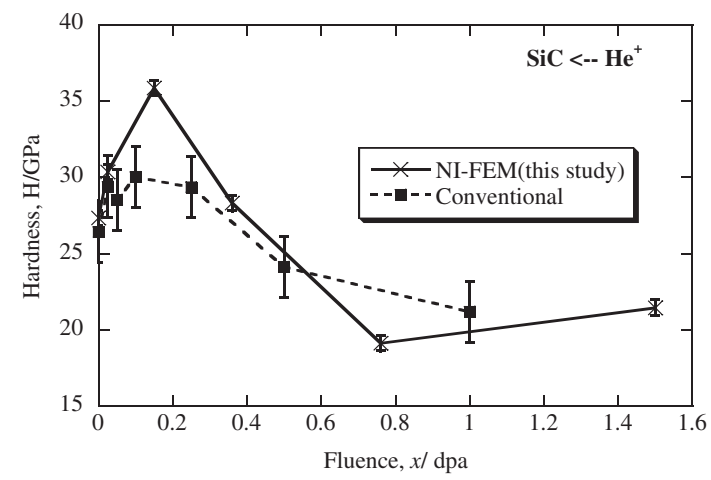

Fig. 3 Variation in hardness of $\alpha$-SiC irradiated by $\mathrm{He}^{+}$. Solid squares ( $\boldsymbol{\square}$ ) represent the hardness estimated by the conventional method, while crosses $(x)$ represent the hardness of the damaged layer determined by the present NI-FEM method.

model as functions of $\mathrm{He}^{+}$-irradiation dose are shown in Fig. 3. The error bars were estimated from the scatter in many measurements (standard deviation) for the conventional method and from the accuracies determined by the interpolation method described in Appendix 1 for the present NI-FEM method. The initial significant hardening is not clearly observed in the conventional method because the method measures the average value of the whole specimen. In addition, one may read the data incorrectly, and interpret them as indicating that the sample is gradually softened on the basis of the magnitude of the error bars.

The changes in $H$ as functions of the irradiation dose, estimated by the present method, are shown in Figs. 4(a)-(c). In all the crystalline samples tested, $H$ increased once at the initial stage of ion irradiation and then gradually decreased with the dose. The irradiation doses at which $H$ starts to decrease in the case of $\mathrm{SiC}$ and $\alpha$-quartz approximately correspond to their reported critical doses for amorphization. $\mathrm{H}^{+}$-irradiated silicon exhibited a marked initial hardening, up to three times the initial $H$ value. Though silicon is not amorphized by $\mathrm{H}^{+}$-irradiation at room temperature, ${ }^{7)} H$ starts to decrease at a dose much lower than the onset of softening of $\mathrm{SiC}$, which is likely ascribable to the termination of broken bonds by hydrogen ( $c f$. Section 5.3).

Figure 4(b) reveals that $H$ of silica glass exhibits a totally different behavior from crystalline $\alpha$-quartz: it initially decreases and then gradually increases with irradiation dose, finally coinciding with the hardness curve of $\alpha$-quartz for more than $1.5 \mathrm{dpa}$. Given that $\alpha$-quartz is amorphized at around $0.2-3 \mathrm{dpa},{ }^{8)}$ the local structure of fused silica glass and amorphous quartz formed by ion irradiation are presumably not the same, but asymptotically approach the same structure by prolonged irradiation. It was reported ${ }^{8)}$ that the density of neutron-irradiated $\alpha$-quartz decreases abruptly at around $0.1 \mathrm{dpa}$ and recovers slightly up to at least $1.1 \mathrm{dpa}$, while neutron-irradiated silica glass densifies gradually and slightly with the dose, finally converging to similar values for more than $1 \mathrm{dpa}$. The $H$ changes shown in Fig. 4(b) correspond well to these reported density changes ( $c f$. Section 5.3). 

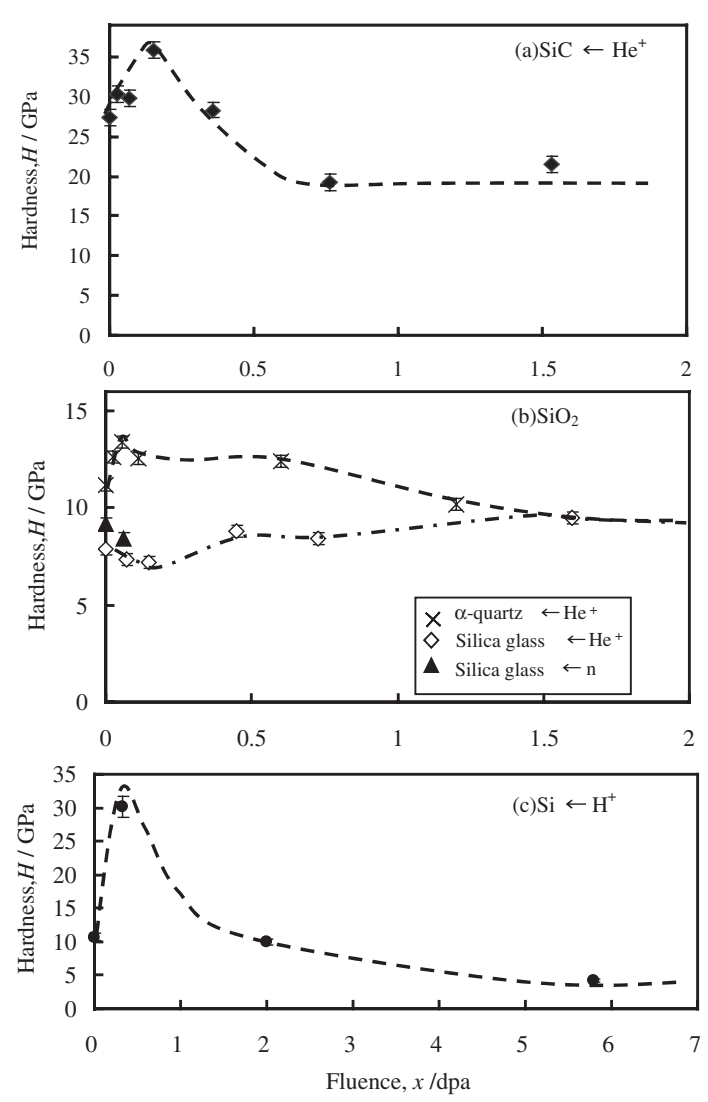

Fig. 4 Variation in hardness with irradiation dose in $\mathrm{He}^{+}$-irradiated $\alpha$-SiC (a), $\mathrm{SiO}_{2}$ ( $\alpha$-quartz and silica glass) (b) and $\mathrm{H}^{+}$-irradiated $\mathrm{Si}$ (c), estimated by the present NI-FEM method. Broken lines are fitting curves based on eq. (1). Solid triangles ( $\boldsymbol{A})$ in (b) represent the experimental data of neutron-irradiated silica glass.

\subsection{Validity of the present multi-layer model}

In this section, we assess the accuracy of the present multilayer analysis in the NI-FEM method. In particular, we might overestimate the $H$ of the topmost layer in the first step, since the crystalline samples initially exhibits marked hardening. We then compare the present results with the $H$ of neutronirradiated silica glasses, in which the defects are distributed uniformly. The results are shown in Fig. 4(b). The neutronirradiated samples are synthesized silica glass and their $H$ 's are slightly different from that of the ion-irradiated sample. Nevertheless, the two types of samples consistently show a similar trend with respect to the initial softening. Another report $^{9)}$ showed that the hardness of neutron-irradiated $\alpha$-SiC changed from $38.7 \pm 2 \mathrm{GPa}$ (initial) to $21.0 \pm 1 \mathrm{GPa}$ (after irradiation to $2.6 \mathrm{dpa}$ ), which is consistent with the present results [Fig. 4(a)].

In the present multi-layer analysis, in each step of which the mechanical parameters of the damaged layer are determined using the values in the previous step, the thickness of the lightly damaged layers in Fig. 2(c) is determined so that the average dpa agrees with that of the damaged layer of the previous step. We then checked whether the parameters obtained were consistent with the values derived by dividing the sample in different manners: Figure 5 compares the values obtained by the present method and those obtained by a four-layer model with layers of equal thickness. In the case of the latter, the values corresponding

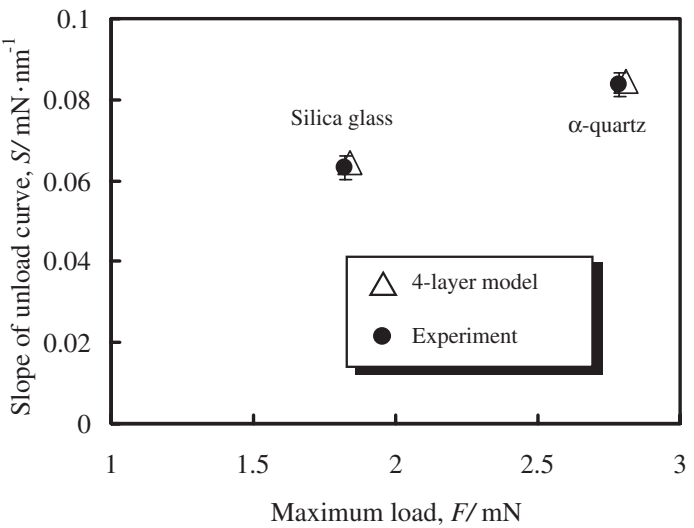

Fig. 5 Comparison between experimental $(F, S)$ and those simulated by the four-layers-with-equal-thickness model, using the data in Fig. 4(b).

to the average dose in Fig. 4 are substituted for the mechanical parameters of each layer, and the ideal L-D curve is simulated. For the present purpose, it is sufficient to compare $F$ and $S$ [Fig. B·1(a)], as shown in Fig. 5. A set of $(F, S)$ values shows good agreement between the initial fourlayer and the equal thickness modeling, which confirmed that the mechanical parameters derived are independent of the layering. Dividing the sample into more than four layers gave similar results within experimental accuracy.

\section{Discussion}

\subsection{Hardness variation in crystalline samples by phenomenological rate equations}

Generally speaking, the mechanical strength (hardness) of metals and alloys increases upon energetic particle irradiation $^{1)}$ because the defects induced hinder the mechanisms whereby plastic deformation occurs, namely dislocation motion and/or slip along the most densely stacked planes. By contrast, plastic deformations of covalent and ionic materials are accompanied by the breaking and re-forming of interatomic bonds, and thus one needs to take into account local destruction phenomena. As explained in Section 4.1, a low concentration of defects increases hardness even in covalent and ionic crystals, but when the defect density exceeds a certain value, they lower the hardness. Meanwhile, silica glass exhibits a complex behavior, suggesting that the plastic deformation mechanisms of crystals and amorphous materials (glass) differ, and hence their mechanical properties cannot be described by one microscopic parameter (e.g., dislocation density). Thus, we introduce the following phenomenological model to semi-quantitatively extract physical parameters characteristic of a variety of materials; it is assumed that hardness of ion-irradiated crystalline materials can be described by the following equations:

$$
\begin{aligned}
& H=C_{1} H_{1}+C_{2} H_{2}+C_{3} H_{3} \\
& C_{1}+C_{2}+C_{3}=1,
\end{aligned}
$$

where $C_{i}$ and $H_{i}(i=1,2,3)$ correspond, respectively, to the concentration and hardness of the crystalline (non-irradiated) phase, hardened phase and amorphous (softened) phase. This model assumes that the variation in hardness with irradiation 
is described by the variation in composition of three components having the constant $H_{i}$ s. In the present model, the experimental hardness values of the non-irradiated and final saturated states can be used for $H_{1}$ and $H_{3}$, respectively. The physical interpretation can be that each component corresponds to a small region having a specific defect density, though their realistic structures should be discussed elsewhere. The validity of eq. (1) is discussed in Appendix 2.

If phase \#1 (non-irradiated phase) subsequently transforms into phase \#2 (hardened phase) and eventually into phase \#3 (softened phase) by displacement damage, the change in the concentration of each phase with irradiation dose (time) is expressed by the following set of ordinary differential equations:

$$
\left\{\begin{array}{l}
\frac{d C_{1}}{d t}=-\phi \sigma_{1} C_{1}+b C_{2} \\
\frac{d C_{2}}{d t}=\phi \sigma_{1} C_{1}-\phi \sigma_{2} C_{2}-b C_{2} \\
\frac{d C_{3}}{d t}=\phi \sigma_{2} C_{2}
\end{array}\right.
$$

where $\phi$ is the ion flux, $\sigma_{1}$ and $\sigma_{2}$ are, respectively, the apparent cross-sections for the transformations from phase \#1 into \#2 and from phase \#2 into \#3, and $b$ is the rate of recovery from phase \#2 to phase \#3. It is assumed that phase \#3 never recovers to phase \#1 or \#2. The solutions to eq. (3) are:

$$
\begin{aligned}
& C_{1}=\frac{1}{2 A}\left\{(A+C) \mathrm{e}^{-\frac{1}{2}(A+B) t}+(A-C) \mathrm{e}^{\frac{1}{2}(A-B) t}\right\} \\
& C_{2}=\frac{1}{2 A}\left\{-(B+C) \mathrm{e}^{-\frac{1}{2}(A+B) t}+(B+C) \mathrm{e}^{\frac{1}{2}(A-B) t}\right\} \\
& C_{3}=\frac{1}{2 A}\left\{-(A-B) \mathrm{e}^{-\frac{1}{2}(A+B) t}-(A+B) \mathrm{e}^{\frac{1}{2}(A-B) t}\right\}+1,
\end{aligned}
$$

where

$$
\begin{aligned}
& A=\sqrt{(a+b+c)^{2}-4 a c,} \\
& B=a+b+c, \\
& C=a-b-c, \\
& a=\sigma_{1} \phi, \\
& c=\sigma_{2} \phi .
\end{aligned}
$$

The parameters $H_{3}, \sigma_{i}(i=1,2)$ and $b$ for each sample were obtained by fitting eqs. (1) and (4)-(11) to the data in Figs. 4(a)-(c), the results of which are tabulated in Table 2. The best-fit curves are shown by solid lines in Fig. 4 .

The concentration change of each component, $C_{i}$ ( $i=$ 1-3), (eqs. (4)-(6)) was plotted as a function of the irradiation dose in Fig. 6, using the derived parameters. One can deduce the following features from Fig. 6 as important information on the plastic deformation mecha-
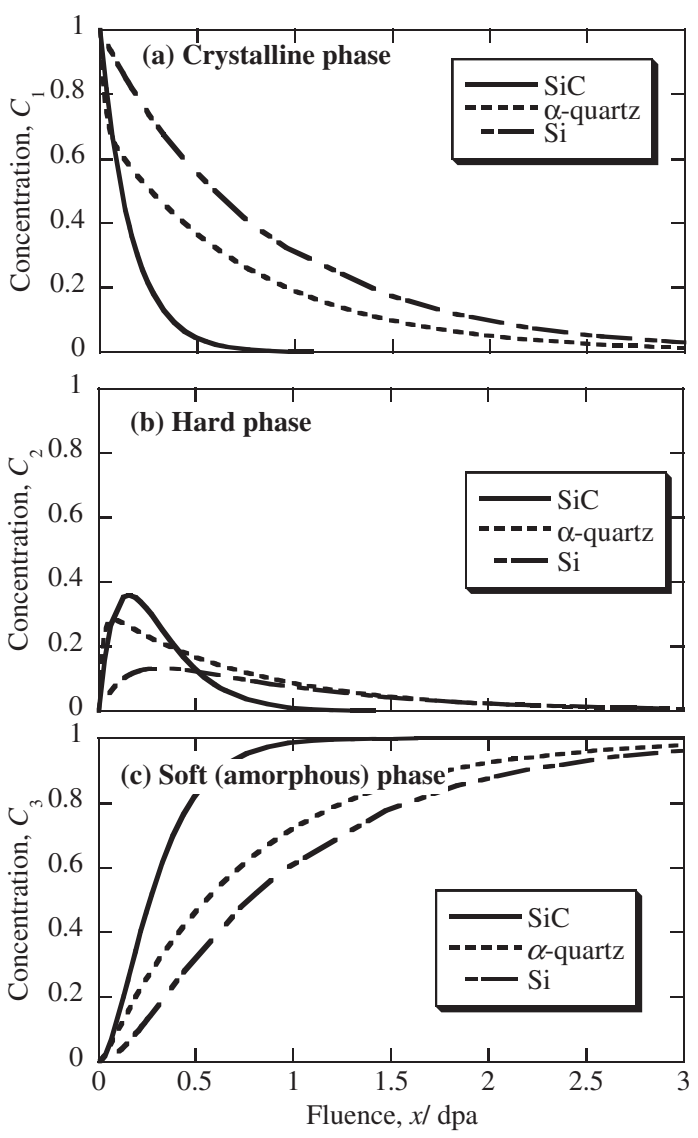

Fig. 6 Changes in $C_{1}$ (a), $C_{2}$ (b) and $C_{3}$ (c) with irradiation dose, derived by the phenomenological rate equations (eqs. (1)-(3)).

nisms: (i) in $\alpha-\mathrm{SiC}$, the irradiation dose, when amorphization is complete $\left(C_{3} \sim 1\right)$, roughly corresponds to the critical dose for amorphization reported by TEM observation. ${ }^{9)}$ (ii) It has been reported that crystalline quartz started to be amorphized at around $0.1 \mathrm{dpa}$ and was fully amorphized at around $1 \mathrm{dpa} .{ }^{8)}$ The present analysis showed that the amorphous component (phase \#3) in quartz slowly increased. This reflects behavior characteristic of $\mathrm{SiO}_{2}$-related materials, which consist of rigid $\mathrm{SiO}_{4}$ tetrahedra interconnected via flexible $\mathrm{Si}-\mathrm{O}-\mathrm{Si}$ bonds. This issue is discussed again in Section 5.3. (iii) The softened phase (phase \#3) in H-irradiated silicon also slowly increased. This corresponds to the fact that silicon is not easily amorphized by H-irradiation and assumes a highly defective state, ${ }^{7)}$ which is discussed in Section 5.2. (iv) The irradiation dose at which the concentration of phase \#2 (hard phase) attains its maximum value is significantly less than the amorphization start dose for $\mathrm{SiC}$ and quartz. Hence, this irradiation-induced-hardening is characteristic of crystalline materials ( $c f$. next section)

Table 2 Derived parameters based on the phenomenological rate eqs. (3)-(11).

\begin{tabular}{ccccccc}
\hline Sample & $H_{1}(\mathrm{GPa})$ & $H_{2}(\mathrm{GPa})$ & $H_{3}(\mathrm{GPa})$ & $\sigma_{1}\left(\mathrm{~m}^{-2}\right)$ & $\sigma_{2}\left(\mathrm{~m}^{-2}\right)$ & $\sigma_{\mathrm{TRIM}}\left(\mathrm{m}^{-2}\right)$ \\
\hline $\mathrm{SiC}$ & 27.3 & 50 & 20 & $2.7 \times 10^{-20}$ & $2.9 \times 10^{-20}$ & $2.9 \times 10^{-21}$ \\
$\alpha$-quartz & 11.1 & 19 & 10 & $5.3 \times 10^{-20}$ & $1.2 \times 10^{-21}$ & $2.7 \times 10^{-21}$ \\
$\mathrm{Si}$ & 10.7 & 166 & 4.2 & $7.5 \times 10^{-22}$ & $2.7 \times 10^{-21}$ & $1.4 \times 10^{-2}$ \\
\hline
\end{tabular}


Table 3 Initial increase rate of $C_{2}$ and increment $n$ (atom numbers) of $C_{2}$ per vacancy introduced.

\begin{tabular}{cccc}
\hline Sample & $\mathrm{d} C_{2} / \mathrm{d} t\left(\mathrm{~s}^{-1}\right)$ & $\mathrm{dpa}$ rate $\left(\mathrm{s}^{-1}\right)$ & $n$ \\
\hline $\mathrm{SiC}$ & $3.4 \times 10^{-3}$ & $3.9 \times 10^{-4}$ & 8.7 \\
$\alpha$-quartz & $9.2 \times 10^{-3}$ & $6.6 \times 10^{-5}$ & 18 \\
$\mathrm{Si}$ & $6.6 \times 10^{-3}$ & $3.6 \times 10^{-4}$ & 1.4 \\
\hline
\end{tabular}

\subsection{Initial increase in hardness in crystalline materials}

The increase in $H$ of crystalline materials at the initial stage of irradiation discussed in Section 4.1 suggests that covalent and ionic materials can be plastically deformed through local dislocation motion, which is hindered by the defects generated. On the basis of the increase in $C_{2}$ per nominal damage rate (dpa rate), we can now estimate the number of phase \#1 (crystalline phase) atoms that were converted into the hard phase (phase \#2). The rate of increase of $C_{2}$ for small $t$ is expressed from eq. (5) as:

$$
\frac{d C_{2}}{d t} \cong \frac{B+C}{2} \text {. }
$$

The value $n$ obtained by dividing $\mathrm{d} C_{2} / \mathrm{d} t$ by the apparent dpa rate is listed in Table 3 for each crystalline sample. $n$ is equivalent to $\sigma_{1} / \sigma_{\text {TRIM }}$ in Table 2 . The remarkable hardening of $\mathrm{H}^{+}$-irradiated silicon $\left(H_{2} / H_{1} \sim 16\right)$ for a single displacement damage (vacancy) induced is localized within the region delimited by the nearest neighbor distance, since $n \sim 1-2$. By contrast, the region delimited by the second neighbor distance $(n \sim 10)$ is presumably hardened in $\mathrm{He}^{+}$. irradiated silicon carbide and quartz, though the increase in hardness is more moderate $\left(H_{2} / H_{1} \sim 2\right)$. These findings can be interpreted as follows: (i) in crystalline silicon, displaced interstitial atoms promptly move away to sinks such as dislocations and surfaces, and residual vacancies with broken bonds terminated by implanted hydrogen atoms act as efficient obstacles against plastic deformation. Since one vacancy can absorb up to 4 hydrogen atoms, the rate of increase in $C_{2}$ is less sensitive to the number of implanted hydrogen atoms. (ii) In silicon carbide, most of the interstitial atoms do not migrate a long distance, but rather, stay around the displaced positions to reconstruct the unsaturated bonds with the surrounding atoms. ${ }^{10)}$ The associated lattice distortions range as far as the second neighbor distance. (iii) The plastic distortion of quartz occurs through bending of the flexible bonds linking the rigid $\mathrm{SiO}_{4}$ tetrahedra, ${ }^{11)}$ which inevitably renders $n$ relatively large.

A similar discussion holds for the $C_{3}$ component. The ratio $\sigma_{2} / \sigma_{\text {TRIM }}$ is larger for $\mathrm{H}^{+}$-irradiated silicon than it is for the others, and hence $C_{3}$ changes by a larger structural unit for defect generation. This is due to the fact that the softened phase \#3 consists of hydrogen-terminated atomic clusters of $\sim 1 \mathrm{~nm}$ in size and is deformed by shear between the clusters. ${ }^{12)}$ In contrast, the deformation in the amorphous structure proceeds via local rearrangements of atomic configurations, as will be discussed in Section 5.4.

\subsection{Hardness change during the later stages of irradi- ation}

Gao et al. ${ }^{13)}$ proposed a theoretical formula that univer- sally predicts the Vickers hardness, $H_{V}$, of covalent (and ionic) materials by assuming that $H_{V}$ is proportional to the number of broken bonds contained in the impression by the Vickers indenter:

$$
H_{V}=C N_{a} E_{g},
$$

where $N_{a}$ is the number of covalent bonds per unit area, $E_{g}$ is the band gap in which the ionic heteropolar component is eliminated and $C$ is the constant of proportionality. The constant $C$ is determined as a function of scaled ionicity of chemical bonds in materials by fitting eq. (13) to the plot of experimental hardness $v s$. ionicity for many covalent materials. The resultant equation predicts the hardness of most covalent materials, though it significantly underestimates the hardness of $\mathrm{SiO}_{2}{ }^{13)}$ This is due to the structure of $\mathrm{SiO}_{2}$, which is made up of $\mathrm{SiO}_{4}$ tetrahedra. These tetrahedra are linked only at their corners and can easily tilt. Plastic deformation is characterized by the rotation and bending of the framework tetrahedra about their shared oxygen atoms, rather than breaking of the bonds of the framework. ${ }^{11)}$

The decrease in hardness of crystalline materials during the later stage of irradiation is presumably no longer structure-dependent. Rather, it is thought to correspond to the decrease in the number density of interatomic bonds due to the displacement damage. Thus, we estimate the irradiation effect on hardness via eq. (13).

It is assumed that $E_{g}$, ionicity, and the proportionality coefficient, $C$, are independent of irradiation damage. Denoting the number of bonds in the unit cell for a constituent element $i$ as $m_{i}$, and the volume of the unit cell as $V$, eq. (13) can be rewritten as

$$
H_{V}=C E_{g}\left(\sum_{i} \frac{m_{i}}{2 V}\right)^{\frac{2}{3}},
$$

where $m_{i}$ and $V$ vary with irradiation. Ion irradiation is thought to break a fraction, $f$, of the interatomic bonds, and $m_{i}$ can be expressed as a function of irradiation time in the following form:

$$
m_{i}(t)=m_{i}(0)[1-f(t)] .
$$

$f$ obeys the following equation: $\left.{ }^{14}\right)$

$$
\frac{d f}{d t}=\phi \sigma(1-f)-k f
$$

where $\phi$ is the ion flux, $\sigma$ is the cross-section for the displacement damage and $k$ is the recovery rate of the broken bonds per unit time. Solving eq. (16) with the initial condition $f(0)=0$ and substituting the solution into eq. (15), we obtain

$$
m_{i}(t)=m_{i}(0) \cdot \frac{k+\phi \sigma e^{-(\phi \sigma+k) t}}{\phi \sigma+k} .
$$

From eqs. (14) and (17), the variation in hardness with irradiation is given by:

$$
H_{V}(t)=H_{V}(0) \cdot \frac{k+\phi \sigma e^{-(\phi \sigma+k) t}}{\phi \sigma+k} \cdot \frac{V(0)}{V(t)} .
$$

Since Vickers hardness gives nearly the same value as the hardness obtained in the present study by a Berkovich 


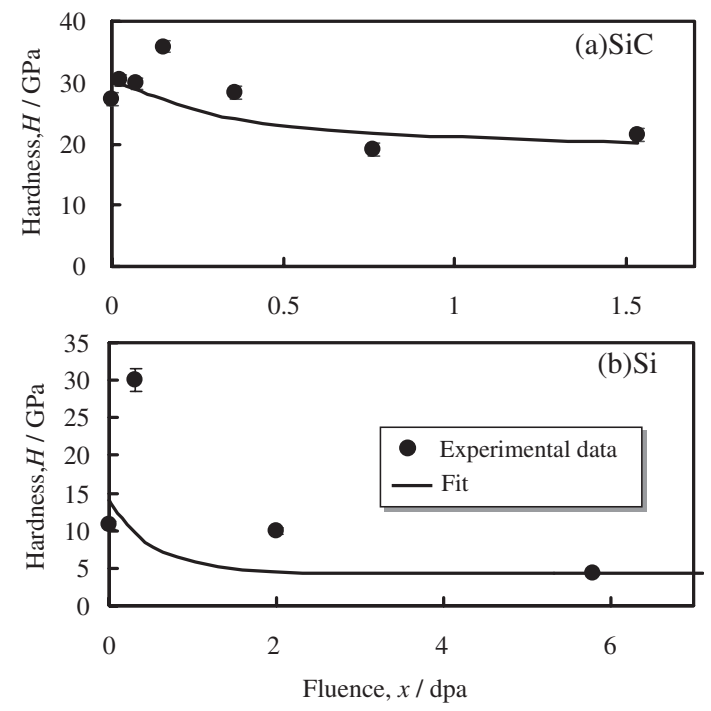

Fig. 7 The fit between the experimental hardness decay and eq. (18) derived from the theoretical consideration of hardness of covalent materials by Gao et al. ${ }^{13)}$

Table 4 Parameters for the later stage of irradiation, derived by eq. (18).

\begin{tabular}{cccc}
\hline Sample & $\sigma_{\text {Gao }}\left(\mathrm{m}^{-2}\right)$ & $k\left(\mathrm{~s}^{-1}\right)$ & $r$ \\
\hline $\mathrm{SiC}$ & $5 \times 10^{-21}(3)$ & $8 \times 10^{-4}(5)$ & $0.6(2)$ \\
$\mathrm{Si}$ & $2 \times 10^{-22}(1)$ & $5 \times 10^{-6}(3)$ & $0.2(1)$ \\
\hline
\end{tabular}

indenter, one can estimate $\sigma$ and $k$ by fitting the experimental results shown in Figs. 4(c) and (a) for silicon and silicon carbide, respectively, with eq. (18). The present formulation, however, does not take the initial hardening into account, and the curve fitting was carried out for the points for the zero doses and the later softening regime. It is known that silicon carbide exhibits considerable volume swelling upon irradiation. We then estimated $V(t)$ from the change in plasmon energy reflecting the valence electron density measured by electron energy-loss spectroscopy. ${ }^{10)}$ For silicon, we took the volume as constant with irradiation.

The best-fit curves are shown in Fig. 7 and the derived parameters are tabulated in Table 4. The parameter $r$ in the table is the apparent final fraction of residual interatomic bonds after the completion of amorphization, and is given by:

$$
r=\frac{\sum_{i} m_{i}(\infty)}{\sum_{i} m_{i}(0)}=\frac{k}{\phi \sigma+k} \text {. }
$$

As shown in Tables 2 and 4, the apparent cross-sections derived are, within the accuracy of the present measurements, in agreement with the estimates obtained by the SRIM code simulations for nuclear stopping $\left(7^{\text {th }}\right.$ column in Table 2 ). This suggests that the softening regime is mainly ascribable to the displacement damage induced by nuclear bombardment. The damage recovery rate, $k$, reflects the effect of hydrogen that terminates broken bonds and hampers the damage recovery, compared to the case of $\mathrm{He}^{+}$-irradiation. The bond survival rate, $r$, also demonstrates the effect of hydrogen, and the value $r=0.2$ for silicon roughly agrees with the upper limit when hydrogen is implanted into silicon. ${ }^{12)}$

The complex behavior of hardness in $\alpha$-quartz and silica glass are beyond the present formulation, though we already discussed the residual part characteristic of $\mathrm{SiO}_{2}$ polytypes, other than the normal part expressed by eq. (18). ${ }^{15)}$

\subsection{Microscopic mechanism for plastic deformation in amorphous structures}

To understand the atomistic mechanism of plastic deformation in amorphous structures under pressure, model simulations based on classical molecular dynamics (MD) were carried out.

An MD cell consisting of $5 \times 5 \times 5$ crystalline silicon unit cells (number of atoms: 1,000; lattice constant: $2.75 \mathrm{~nm}$ ) was prepared; the MD cell was melted by keeping it at 4,000 $\mathrm{K}$ for $40 \mathrm{ps}$, followed by quenching to $300 \mathrm{~K}$ at a cooling rate of $100 \mathrm{~K} / \mathrm{ps}$ to obtain an amorphous silicon structure with the cubic cell shape retained. The MD simulations were performed under NTP ensemble with the Tersoff interatomic potential $^{16)}$ and the periodic boundary condition imposed. A uniaxial compressive stress of $0.8-2 \mathrm{GPa}$ was applied for 80 ps to the resultant amorphous MD cell (lattice constant: $2.65 \mathrm{~nm}$ ) and the time dependence of the lattice constants and atomic positions was examined.

Projected atomic trajectories and the changes in the lattice parameters are shown in Figs. 8 and 9, respectively, for the crystalline and amorphous MD cells under a uniaxial stress in the $z$-direction at $300 \mathrm{~K}$. For the crystalline cell, the tetragonal distortion and the applied stress immediately balanced elastically and reached a steady state. For the amorphous cell, on the other hand, the MD cell was distorted into an orthorhombic shape, and the lattice parameters of the MD cell continually changed with time even at low stress, and did not reach a steady state even after $80 \mathrm{ps.} \mathrm{The}$ deformation was retained when the stress was released and hence the deformation was plastic. The atom trajectories for the amorphous case show that each atom is displaced by a similar distance, in contrast to the crystalline case. Furthermore, the radial distribution function (not shown) was unaltered by the applied stress. It can thus be said that amorphous structures can be deformed by the constituent atoms collectively rearranging their positions so as to leave the total cohesive energy unchanged. This deformation
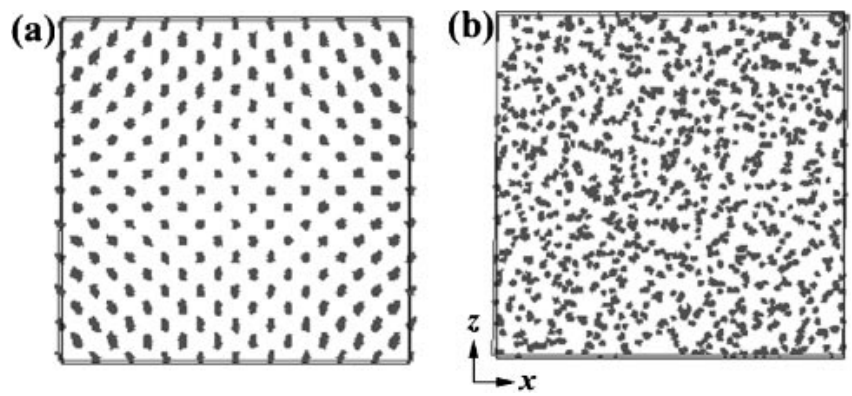

Fig. 8 Projected atom trajectories in the MD simulations. The black lines delineate the initial MD cell shape and the grey lines represent the final shape. (a) Crystalline silicon, $2 \mathrm{GPa}$ compressive stress in the $z$-direction at $300 \mathrm{~K}$. (b) Amorphous silicon, $800 \mathrm{MPa}$ at $300 \mathrm{~K}$. 

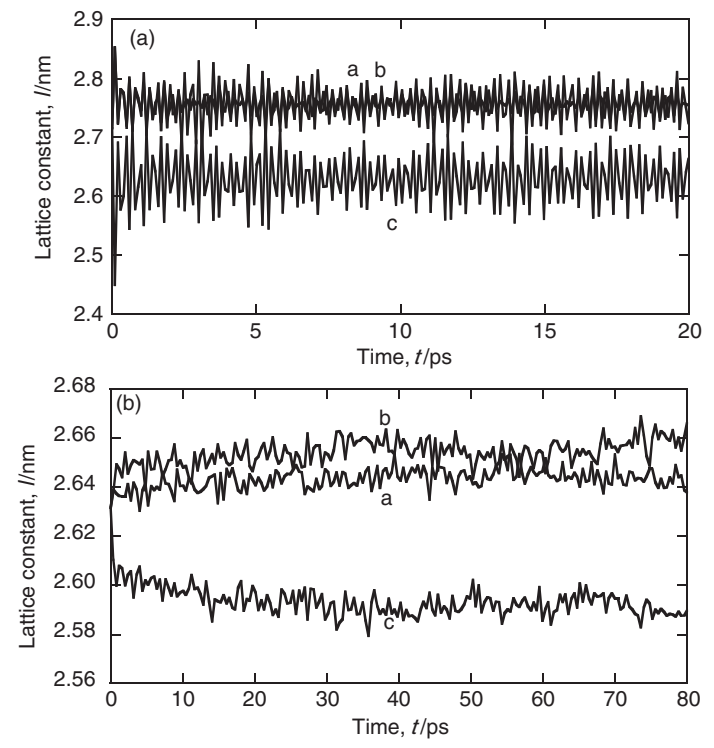

Fig. 9 Time evolution of lattice parameters of the MD cell. (a) and (b) correspond to the cases in Figs. 8(a) and (b), respectively.

mechanism is only possible for amorphous structures having no translational symmetry. Unlike the case for crystalline structures, amorphous structures need not maintain local translational symmetry and hence the change in structural freedom in response to the external force can be described by linear combinations of bond breaking effects, as discussed in the previous section. The above discussion is to be qualitatively examined at further length.

It was reported that silicon was not amorphized by $\mathrm{H}^{+}$ irradiation at doses below $10 \mathrm{dpa}$, though a similar deformation mechanism works with $\mathrm{H}$-terminated small atom clusters acting as unit-building blocks. ${ }^{12)}$

\section{Conclusions}

We developed a method for estimating the mechanical properties of ion-irradiated samples by applying a finite element method to nanoindentation tests and multi-layer modeling of ion-irradiated samples. The present method enabled us to obtain the mechanical parameters of the damaged layer embedded in the samples with higher accuracy. We applied the method to $\mathrm{He}^{+}$-irradiated silicon carbide, $\alpha$-quartz single crystals and silica glass, and an $\mathrm{H}^{+}$irradiated silicon single crystal. The results are summarized as follows:

(1) Hardness of crystalline materials increased for doses up to $0.1-0.2 \mathrm{dpa}$. This suggested that plastic deformation of crystalline ceramics is induced by dislocation motion, as in metals and alloys.

(2) Hardness of crystalline materials decreased upon prolonged irradiation and asymptotically approached a certain value as a result of amorphization. This behavior can be formulated and semi-quantitatively explained on the basis of the change in the number density of interatomic bonds due to irradiation-induced bond breakage.

(3) Hardness of silica glass (amorphous) and irradiation- induced amorphous $\mathrm{SiO}_{2}$ differed significantly, suggesting that their local structures are different. Hardness of the two structures eventually converged to the same value upon heavy irradiation.

(4) Plastic deformation of amorphous structures proceeds slowly and continuously by collective rearrangement of the local atomic configurations to minimize the effect of the external stress. The deformation behavior resembles creep phenomena, though it could be specific to amorphous structures.

(5) The present model described in (2) and (3) above does not explain the mechanical properties of $\mathrm{SiO}_{2}$ polytypes. It is presumably necessary to allow for not only the change in the atom bond density but also the free rotation of $\mathrm{SiO}_{4}$ tetrahedra about the shared oxygen atoms.

The analyses based on phenomenological rate equations developed in the present study can apply widely to covalent ceramics, thus providing a fundamental framework for discussing quantitatively how irradiation damage changes their mechanical properties by comparing the extracted materials parameters. The materials parameters obtained still need to be discussed separately in terms of the atomic-scale mechanisms involved.

\section{Acknowledgments}

We are very grateful to Dr. N. Huber of Forschungszentrum Karlsruhe and Dr. J. Knapp of Sandia National Laboratories for giving us much invaluable advice during the development of the FEM simulation code for the nanoindentation test. We also thank Professor M. Sakai of Toyohashi University of Technology for useful discussions on the estimation of the mechanical properties of materials by nanoindentation. The neutron-irradiated silica glass samples were kindly supplied by Professor M. Hasegawa of Institute for Materials Research, Tohoku University. This work was partly supported by Grants-in-Aid for Scientific Research (A) and (B) of JSPS, and for 21 century COE program (Isotope Science and Engineering from Basics to Applications) of MEXT.

\section{Appendix A: Outline of nanoindentation-FEM (NI- FEM) simulation ${ }^{3)}$}

FEM modeling of nanoindentation tests was performed using the multi-purpose FEM analysis code ABAQUS/ Standard Ver. $6 .{ }^{17)}$ We adopted a two-dimensional cylindrical symmetry model in the nanoindentation simulation to reduce computational time. In the computational model, the Berkovich indenter was thus replaced by a conical indenter with the same projected contact area. The indenter movement was controlled by displacing the elements on the indenter surface, and a theoretical L-D curve was obtained by taking the reaction force and displacement of the reference node of the indenter surface as the load and displacement, respectively.

The hardness, $H$, measured by nanoindentation is defined in terms of the maximum load, $F$, and the projected contact area, $A$, referring to the $\mathrm{L}-\mathrm{D}$ curve in Fig. A.1:2) 


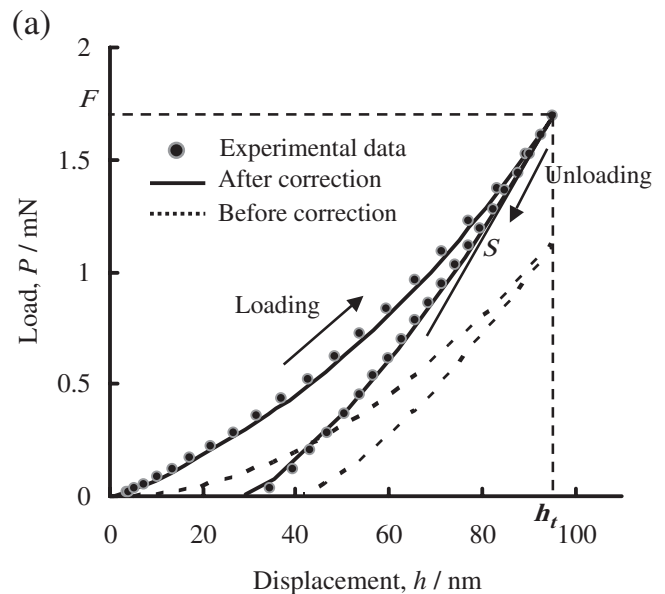

(b)

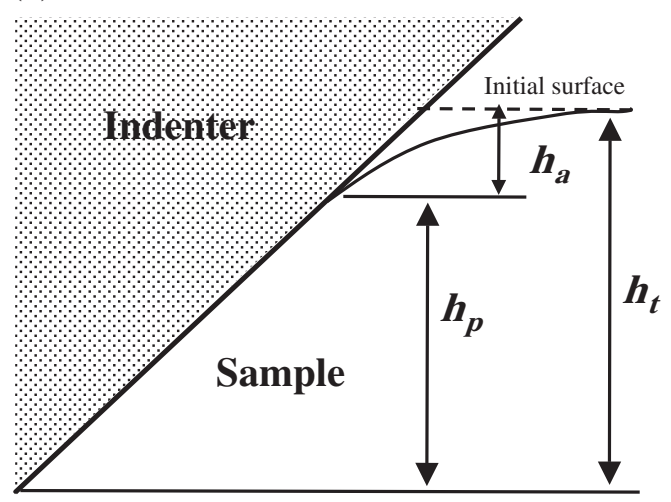

Fig. A.1 Representative experimental and theoretical (simulated) L-D curves.

$$
H=\frac{F}{A} .
$$

The nanoindentation instrument is usually assumed to be a rigid body, though in practice, the elastic response of the instrument itself does influence the results under the minute loads and displacements typically used in nanoindentation tests. The diamond indenter is not ideally sharp at the nanometer scale and its bluntness affects the results significantly, particularly for small indentation depths. Experimental and simulated L-D curves for a standard sample (silica glass) with known mechanical parameters $(E, Y)$ are compared in Fig. A.1. In the present study, all the above instrument-related effects were incorporated into the indenter shape effect by expanding the corrected projected contact area of the indenter into a polynomial with $6^{\text {th }}$ terms. ${ }^{3)}$ This correction resulted in a nearly perfect match between the experimental and simulated L-D curves, as shown in Fig. A·1.

For samples with unknown $(E, Y)$, the computer simulation is repeated until the simulated $\mathrm{L}-\mathrm{D}$ curve agrees with the experimental one. The interpolation method introduced in Ref. 3) was then used to let the result converge efficiently. The final accuracies of the mechanical parameters were estimated from the standard deviations of $F$ and $S$, and the error propagation formulae obtained from the interpolation equations.

We are able to draw an ideal L-D curve using a computer

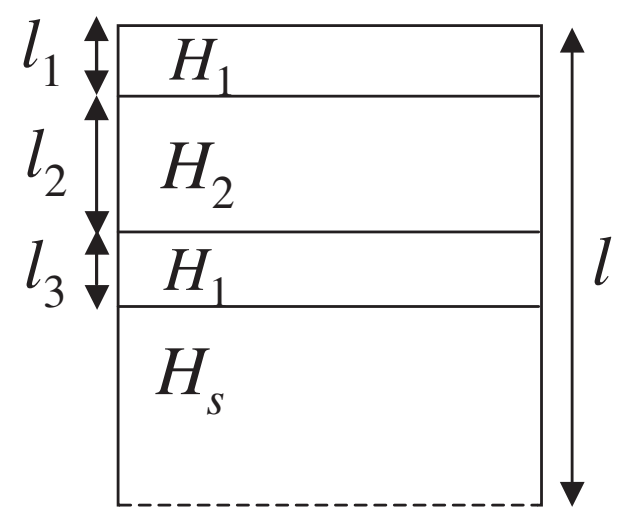

Fig. B.1 Schematic of the 4-layer model to test eqs. (B.3) and (B.4). The total thickness, $l$, is the net thickness affecting the sample hardness to be determined.

for a bulk sample having a set of obtained parameters $(E, Y)$ and calculate the hardness $H$ accurately via eq. (A·1).

\section{Appendix B: Justification of eq. (1) in the multi-layer model}

A number of mechanical models have been proposed for the hardness of an elasto-plastic body consisting of multicomponents. Jonsson and Hogmark ${ }^{18)}$ proposed an area law of mixtures so that the hardness of the film can be extracted from the hardness of the film-substrate combination, weighing the relative area of the contact carried by film and substrate. Sakai ${ }^{19)}$ proposed the energy principle, in which the hardness is proportional to the inverse square of the dissipated energy associated with deformation. We then examined which of the following two is appropriate for the present multi-layer model:

$$
\begin{aligned}
& H_{t}=\sum_{i} C_{i} H_{i} \\
& \frac{1}{\sqrt{H_{t}}}=\sum_{i} \frac{C_{i}}{\sqrt{H_{i}}} .
\end{aligned}
$$

In the case of the multi-layer model shown in Fig. B.1, the total hardness $H_{t}$ directly measured in a nanoindentation test should be the hardness of the combination from the surface to depth $l$, and $l$ usually ranges from several to ten times the maximum indentation depth. Since $H_{t}$ and $H_{i}$ of each layer having a thickness of $l_{i}$ was determined in the present study, eqs. (B·1) and (B-2) are rewritten in terms of $l$ :

$$
\begin{aligned}
H_{t} & =\sum_{i} l_{i} H_{i}+\left(l-\sum_{i} l_{i}\right) H_{u} \\
\frac{1}{\sqrt{H_{t}}} & =\sum_{i} \frac{l_{i}}{\sqrt{H_{i}}}+\left(l-\sum_{i} l_{i}\right) \frac{1}{\sqrt{H_{u}}},
\end{aligned}
$$

where $H_{u}$ is the hardness of an unirradiated sample (nondamaged region). We can now estimate $l$ for each irradiation dose, using eq. (B.3) or (B.4). For a given sample, the correct expression should give the same $l$ for different irradiation doses. As a result, eq. (B-3) gave an $l$ value three to five times the maximum indentation depth for all the samples, which 
was accurate to within $5 \%$ for each sample. On the other hand, the other model based on eq. (B.4) gave an overall total hardness predominantly determined by the harder layer. Hence, the linear combination model of eq. (B-1) is suitable for discussing the hardness change in the multi-layer model in Section 5.1. eq. (B.2) yielded no good fit between the theoretical formulae and experimental data in the initial hardening regime.

We conducted a similar analysis for the Young's modulus, $E$, and the yield stress, $Y$, and found that the linear combination model gave a good fit for both parameters.

\section{REFERENCES}

1) Materials Science Society of Japan, ed.: Irradiation Effects in Materials (in Japanese) (Shokabo, Tokyo, 1994) pp. 1-90.

2) A. C. Fischer-Cripps: Nanoindentation, (Springer, New York, 2002) pp. $9-18$.

3) J. A. Knapp, D. M. Follstaedt, S. M. Myers, J. C. Barbour and T. A. Friedmann: J. Appl. Phys. 85 (1999) 1460-1474.
4) W. C. Oliver and G. M. Pharr: J. Mater. Res. 7 (1992) 1564-1583.

5) K. Izumi, K. Yasuda, C. Kinoshita and M. Kutsuwada: J. Nucl. Mater. 258-263 (1998) 1856-1860.

6) http://www.srim.org/SRIM/SRIM2003.htm

7) S. Muto, S. Takeda and M. Hirata: Mater. Sci. Forum 196-201 (1995) 1171-1176.

8) M. Hasegawa, M. Saneyasu, M. Tabata, Z. Tang, Y. Nagai, T. Chiba and Y. Ito: Nucl. Instr. Meth. Phys. Res. B 166-167 (2000) 431-439.

9) L. L. Snead and J. C. Hay: J. Nucl. Mater. 273 (1999) 213-220.

10) S. Muto and T. Tanabe: J. Appl. Phys. 93 (2003) 3765-3775.

11) I. J. McColm: Ceramic Hardness (Plenum, London, 1990).

12) S. Muto, T. Tanabe and S. Igarashi: Physica Scr. T108 (2004) 19-22.

13) F. Gao, J. He, E. Wu, S. Liu, D. Yu, D. Li, S. Zhang and Y. Tian: Phys. Rev. Lett. 91 (2003) 015502.

14) W. J. Weber and L. M. Wang: Nucl. Instr. Meth. B 106 (1995) 298302 .

15) S. Nakano, S. Muto and T. Tanabe: Mater. Sci. Eng. 400-401 (2005) 382-385.

16) J. Tersoff: Phys. Rev. B 49 (1994) 16349-16352.

17) http://www.abaqus.com/

18) B. Jonsson and S. Hogmark: Thin Solid Films 114 (1984) 257-269.

19) M. Sakai: Acta Metall. Mater. 41 (1993) 1751-1758. 